\title{
THE DECOMPOSITION OF THE GENERAL COLLINEATION OF SPACE INTO THREE SKEW REFLECTIONS*
}

BY

\section{EDWIN B. WILSON}

A number of years ago several investigators $\dagger$ published independently this theorem: Any screw motion-the most general mechanical motion of space - can be decomposed into the product of two semi-rotations. By a semi-rotation is meant that transformation which consists of rotating space through $180^{\circ}$ about a fixed axis $l$. For generalizing however it is more convenient to look at this transformation as reflection in a line. From this point of view a semirotation may be defined as that transformation which replaces each point $P$ of space by a point $P^{\prime}$ such that the line $P P^{\prime}$ cuts orthogonally a fixed line $l$ and is bisected by it.

If we turn to the projective group of three dimensions we find in it, as a transformation corresponding to the semi-rotation of the mechanical group, the skew reflection which may be defined as follows: $A$ skew reflection is that transformation of space which replaces each point $P$ by a point $P^{\prime}$ such that the line $P P^{\prime}$ cuts each of two non-coplanar lines $l, l^{\prime}$ and is divided harmonically by them. The lines $l, l^{\prime}$ are called directrices.

The purpose of this paper is to ask and answer the question: Is it possible to decompose the general collineation of space into the product of a number of skew reflections; and if so, what is the least number of skew reflections involved in such a decomposition? $\ddagger$ The question will be answered by giving

\footnotetext{
* Presented to the Society October 28, 1899. Received for publication November 18, 1899.

† Halphen, Nouvelles Annales de Mathématiques, 3d series, vol. 1, p. 296, 1882. Burnside, On the Resultant of Two Finite Displacements of a Rigid Body; Messenger of Mathematics, vol. 19, p. 104, 1889.

Wimnkr, Die Zusammensetzung zweier endlicher Schraubungen zu einer einzigen; Berichte d. Gesellschaft d. Wissenschaften zu Leipzig, vol. 42, p. 13, 1890.

$\ddagger$ After writing this paper I was informed of an article by M. X. ANTOMARI entitled Sur un cas particulier de la transformation homographique in the Nouvelles Annales de Mathématiques, Nov., 1898, p. 489 . In this paper M. ANTOMARI among other things decomposes the general collineation into the product of three special transformations which may be defined as follows : Each point $P$ of space goes over into a point $P^{\prime}$ such that the line $P P^{\prime}$ cuts two given lines $l, l^{\prime}$ and is divided by them in a given constant cross-ratio. One sees that this transformation is more general than the skew reflection (in which the given constant cross-ratio is - 1), and that it is not involutory. For these reasons the decomposition given by M. ANTOMARI is apparently of less interest and importance than the decomposition of this paper.
} 
a geometric construction for the skew reflections whose product is the given collineation.

For purposes of proof one needs a number of preliminary propositions, corollaries of the definition of skew reflection.

I. Any line $p$ cutting the two directrices $l$ and $l^{\prime}$ of a skew reflection is transformed into itself.

II. The transformation of the points on $p$ is such that any point $P$ is replaced by such a point $P^{\prime}$ that $P$ and $P^{\prime}$ divide harmonically the intersections of $p$ with $l$ and $l^{\prime}$.

III. If a line $p$ is transformed into itself it must cut both directrices, or coincide with one of them.

IV. Any skew reflection whose directrices cut harmonically two opposite edges of a tetrahedron merely interchanges the vertices and leaves the tetrahedron itself invariant.

V. If a skew reflection leaves a tetrahedron invariant and neither directrix contains a vertex of the tetrahedron, then the directrices cut harmonically two opposite edges of the tetrahedron.

Lemma.-There are $\infty^{2}$ skew reflections which interchange the vertices $A$ and $C, B$ and $D$ of a given tetrahedron $A B C D$, leaving the tetrahedron itself invariant.

For the two points of intersection of one of the directrices with two the opposite edges $A C$ and $B D$ of the tetrahedron may be chosen arbitrarily. The points of intersection of the other directrix with those edges are then uniquely determined by the harmonic property (IV). It is now evident that any given point $P$ of space will be transformed into a point $P^{\prime}$, which will probably vary and describe a two-dimensional locus as the directrices of the skew-reflections, which leave the given tetrahedron invariant, take on their $\infty^{2}$ different positions.

TheOREM.-The locus of the point $P^{\prime}$, into which a given general point $P$ is carried by the $\infty^{2}$ skew reflections whose directrices cut harmonically two given opposite edges of a tetrahedron, is a quadric surface $Q$ passing through and determined by the other four edges of the tetrahedron and the given point $P$.

Let $A B C D$ be the given tetrahedron; $A C$ and $B D$, its given opposite edges. We choose $A B C D$ as the coördinate tetrahedron $x_{1} x_{2} x_{3} x_{4}$. Let the directrix $l$ meet $A C$ in the point $\left(c_{1}, 0, c_{3}, 0\right)$ and $B D$ in the point $\left(0, c_{2}, 0, c_{4}\right)$, where $c_{1} c_{2} c_{3} c_{4} \neq 0$; the directrix $l^{\prime}$ then meets $A C$ in the point $\left(c_{1}, 0,-c_{3}, 0\right)$ and $B D$ in the point $\left(0, c_{2}, 0,-c_{4}\right)$. The skew reflection with directrices $l, l^{\prime}$ transforms a point $P$ with the coördinates $\left(y_{1}, y_{2}, y_{3}, y_{4}\right)$ into the point $P^{\prime}$ with the coördinates $\left(y_{1}^{\prime}, y_{2}^{\prime}, y_{3}^{\prime}, y_{4}^{\prime}\right)$, where

$$
\rho y_{1}^{\prime}=\frac{c_{1}}{c_{3}} y_{3}, \quad \rho y_{2}^{\prime}=\frac{c_{2}}{c_{4}} y_{4}, \quad \rho y_{3}^{\prime}=\frac{c_{3}}{c_{1}} y_{1}, \quad \rho y_{4}^{\prime}=\frac{c_{4}}{c_{2}} y_{2},
$$


as is readily proved. If the quantities $c_{1}, c_{2}, c_{3}, c_{4}$ are considered constant and the $y$ 's as the varying point coördinates, these equations establish the transformation of skew reflection between the points $y$ and the points $y^{\prime}$. If however we take the point $P,=\left(y_{1}, y_{2}, y_{3}, y_{4}\right)$, as any particular given point on no one of the faces of the tetrahedron, by eliminating the $c_{1}, \ldots, c_{4}, \rho$ (considered as variable) we have the locus of the transformed point $P^{\prime},=\left(y_{1}^{\prime}, y_{2}^{\prime}\right.$, $\left.y_{3}^{\prime}, y_{4}^{\prime}\right)$, in the running coördinates $x$ :

$$
x_{1} x_{3} y_{2} y_{4}=x_{2} x_{4} y_{1} y_{3}
$$

which is indeed a quadric surface through the point $P$ and the four edges not cut by $l$ and $l^{\prime}: '$

$$
x_{1}=x_{2}=0, \quad x_{1}=x_{3}=0, \quad x_{2}=x_{3}=0, \quad x_{3}=x_{4}=0 .
$$

Lemma.-Two quadrics $Q$ and $Q^{\prime}$, each of which passes through a different closed contour consisting of four edges of the same tetrahedron, intersect in at least one line which does not lie in any face of the tetrahedron.

Given the tetrahedron $A B C D$. Let $Q$ pass through the contour $A B$, $B C, C D, D A$; and $Q^{\prime}$ through $A C, C B, B D, D A$. Let $P$ be any point on $Q$ and in no face of the tetrahedron, and let $P^{\prime}$ be such a point on $Q^{\prime}$. Draw the line $p$ passing through $P$ and cutting $A B$ and $D C$. The line $p$ has three points common with $Q$ and hence lies entirely in $Q$. In like manner $p^{\prime}$, the line passing through $P^{\prime}$ and cutting $A C$ and $B D$, will lie wholly in the quadric $Q^{\prime}$. Since the three non-intersecting lines $B C, p$, and $D A$ lie in $Q, Q$ may be considered as generated by all lines cutting these three. In like manner $Q^{\prime}$ may be considered as generated by all lines cutting $B C, p^{\prime}$, and $D A$. But there is always at least one line which cuts any four lines. Hence there must be one line which cuts $B C, D A, p$, and $p^{\prime}$, and which consequently lies in both quadrics $Q$ and $Q^{\prime}$. Moreover it can not lie in any of the faces. For if it did, the points $P$ and $P^{\prime}$ would lie in that facewhich is contrary to hypothesis. Hence the lemma is established.

Fundamental Theorem.-A collineation of space with four non-coplanar fixed points $A, B, C$, and $D$ can be decomposed into the product of three skew reflections.*

Let the collineation be determined by its four fixed points $A, B, C, D$ and a pair of corresponding points $P$ and $P^{\prime}$.

In the first take a skew reflection which has directrices cutting harmonically $A C$ and $B D$ and which carries $P$ into some point $\bar{P}$ lying on the intersection

* It is to be noted that all cases of collineations which have more than four non-coplanar fixed points will come under this theorem. The cases where there are less than four require each a special treatment. 
of the quadric $Q$, determined by $P$ and the contour $A B, B C, C D, D A$, with the quadric $Q^{\prime}$ determined by $P^{\prime}$ and the contour $A C, C B, B D, D A$. Then take a skew reflection which has directrices cutting harmonically $A B$ and $D C$ and which carries $\bar{P}$ into $P^{\prime}$. Finally take a skew reflection which has directrices cutting harmonically $B C$ and $D A$ and which leaves $P^{\prime}$ unchanged.

Then the product of these three skew reflections in the order named is the collineation which has $A, B, C, D$ as fixed points and which carries $P$ into $P^{\prime}$. For by the first skew reflection $A, B, C, D$ go over respectively into $C, D, A, B$; and these in turn by the second become respectively $D$, $C, B, A ;$ and these in their turn by the third go over respectively into the original positions $A, B, C, D$. Hence $A, B, C, D$ are fixed points. By the construction also $P$ was carried into $P^{\prime}$. The three skew reflections thus give the required collineation.

That two skew reflections cannot be so chosen as to yield an arbitrarily given collineation may be seen at once from the following theorem, the proof of which involves no difficulty.

Theorem.-The necessary and sufficient condition that a collineation which has four and only four fixed points be resoluble into the product of two skew reflections is that the directrices of each of the two reflections shall cut harmonically one and the same pair of opposite edges of the fixed tetrahedron and that one pair of corresponding points lies on a quadric surface which passes through the other four edges of the tetrahedron.

YALE UNIVERSITY,

October, 1899. 